\title{
Reverse Stability of Oxyluciferin Isomers in Aqueous Solutions
}

\author{
Yoshifumi Noguchi ${ }^{1}$, Miyabi Hiyama $^{1}$, Motoyuki Shiga ${ }^{2}$, Osamu Sugino ${ }^{1}$, and \\ Hidefumi Akiyama ${ }^{1}$ \\ ${ }^{1}$ Institute for Solid State Physics, The University of Tokyo, \\ 5-1-5 Kashiwanoha, Kashiwa, Chiba 277-8581, Japan \\ ${ }^{2}$ Center for Computational Science and E-Systems, Japan Atomic Energy Agency \\ 148-4 Kashiwanoha Campus, 178-4 Wakasiba, Kashiwa, Chiba, 277-0871, Japan \\ E-mail: y.noguchi@issp.u-tokyo.ac.jp
}

\section{CONTENTS}

Table S1. Details of total energies of isolated oxyluciferins in vacuum.

Figure S1. Details of oxyluciferin and water molecules configurations.

Figure S2. Details of atomic charge simulated by natural population analysis.

Figure S3. Numbering for each atom.

Figure S4. Detailed information of initial water molecules configurations.

Figure S5. Initial configurations.

Figure S6. Detailed total energies obtained from MD simulations.

Table S2. List of bond length.

Table S3. List of number of hydrogen bonds.

Figure S7-S10. Details of radial distribution functions and coordination numbers.

Figure S11. Wave functions.

Figure S12-14. Chemical bond distance between oxyluciferin (O) and oxyluciferin $(\mathrm{H})$ and hydrogen bond distance between oxyluciferin $(\mathrm{O})$ and the nearestneighbor water molecule $(\mathrm{H})$

keto.mpg, enol.mpg, and enolate.mpg. Movie files of the trajectory obtained from MD simulations (not attached this PDF file). 
Table S1. Total energies in eV simulated for keto, enol, and enolate in vacuum.

\begin{tabular}{|c|c|c|c|c|c|}
\hline & SVWN & BLYP & B3LYP & HSE1PBE & MP2 \\
\hline keto & 0.00 & 0.00 & 0.00 & 0.00 & 0.00 \\
\hline enol & +0.28 & +0.55 & +0.48 & +0.42 & +0.61 \\
\hline enolate & +0.56 & +0.85 & +0.85 & +0.75 & +1.10 \\
\hline
\end{tabular}



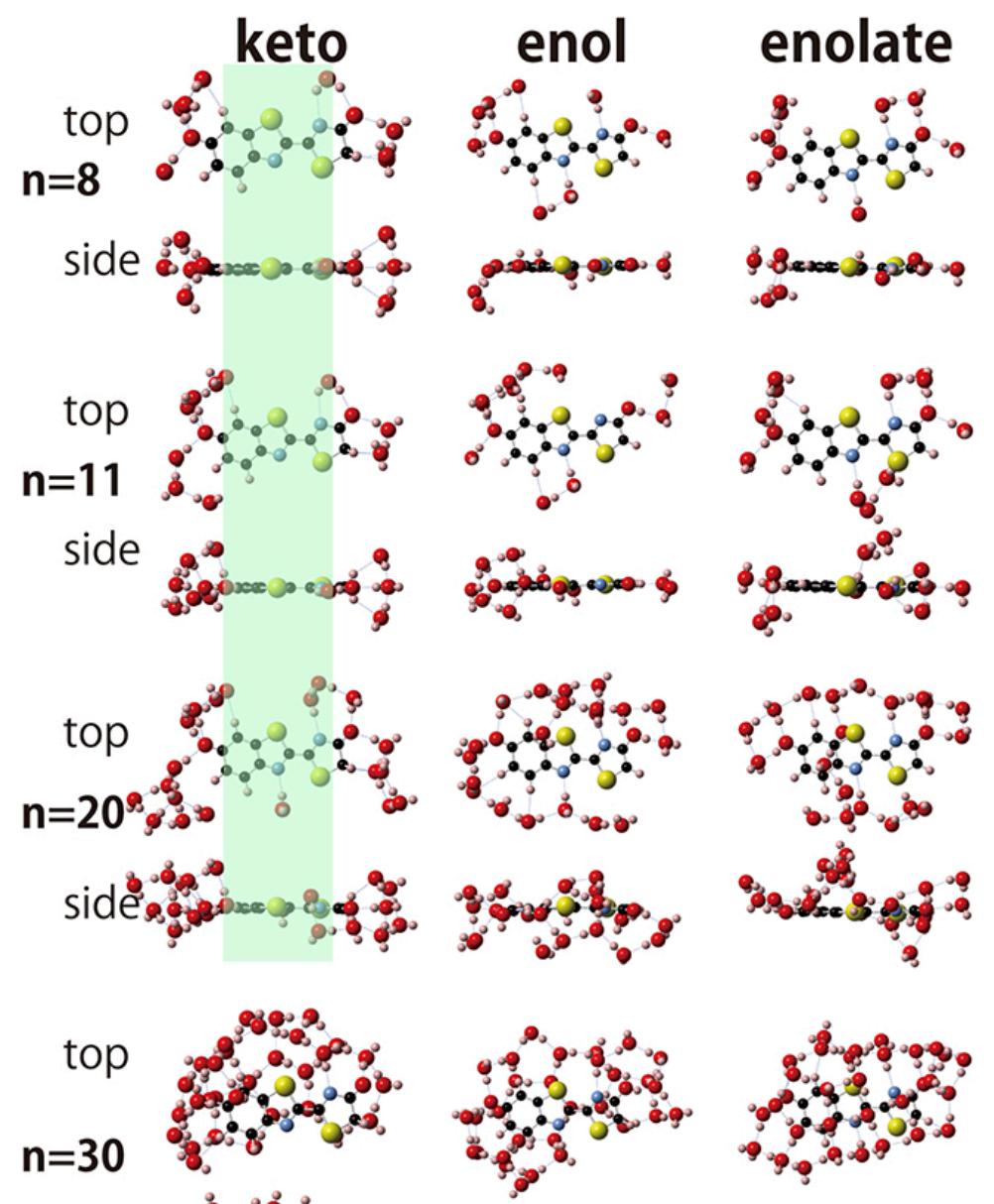

side 8.8
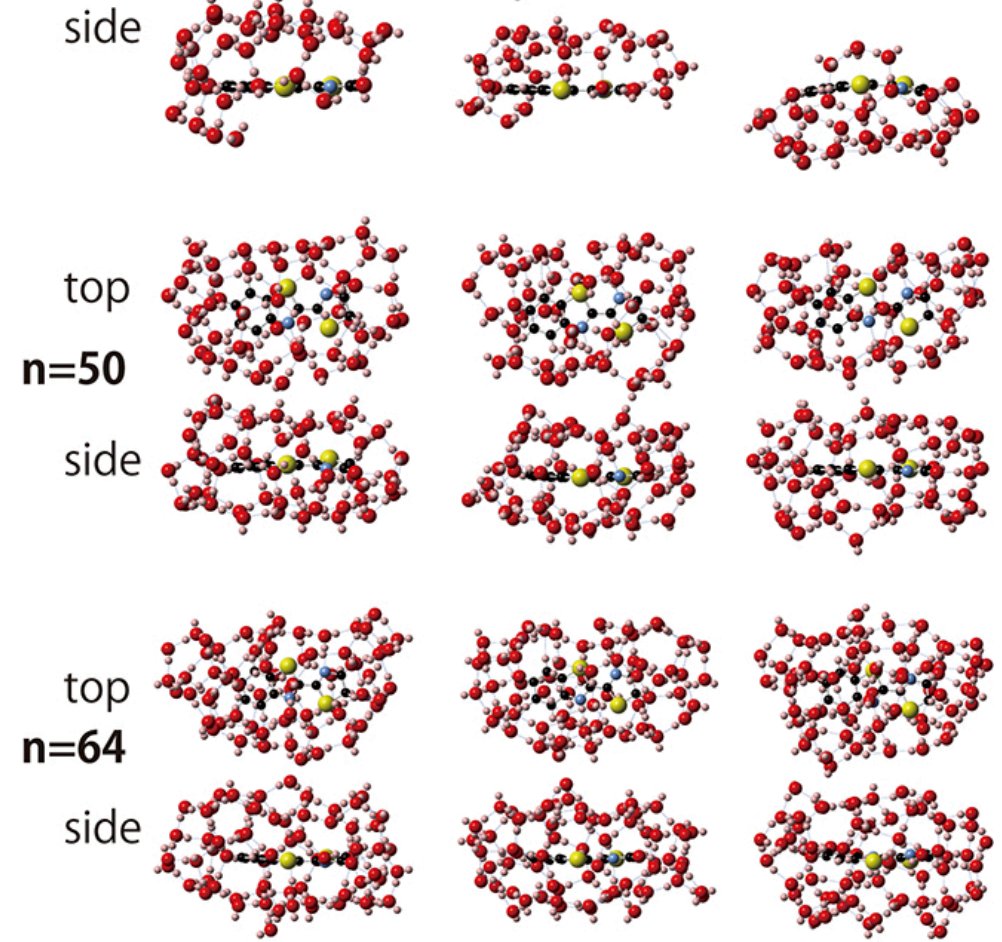

Figure S1. Atomic geometries of oxyluciferin and $\left(\mathrm{H}_{2} \mathrm{O}\right)_{n}$ (where $n=8,11,20,30,50$, and 64) optimized by BLYP/6-311G* together with PCM located outside the region. 

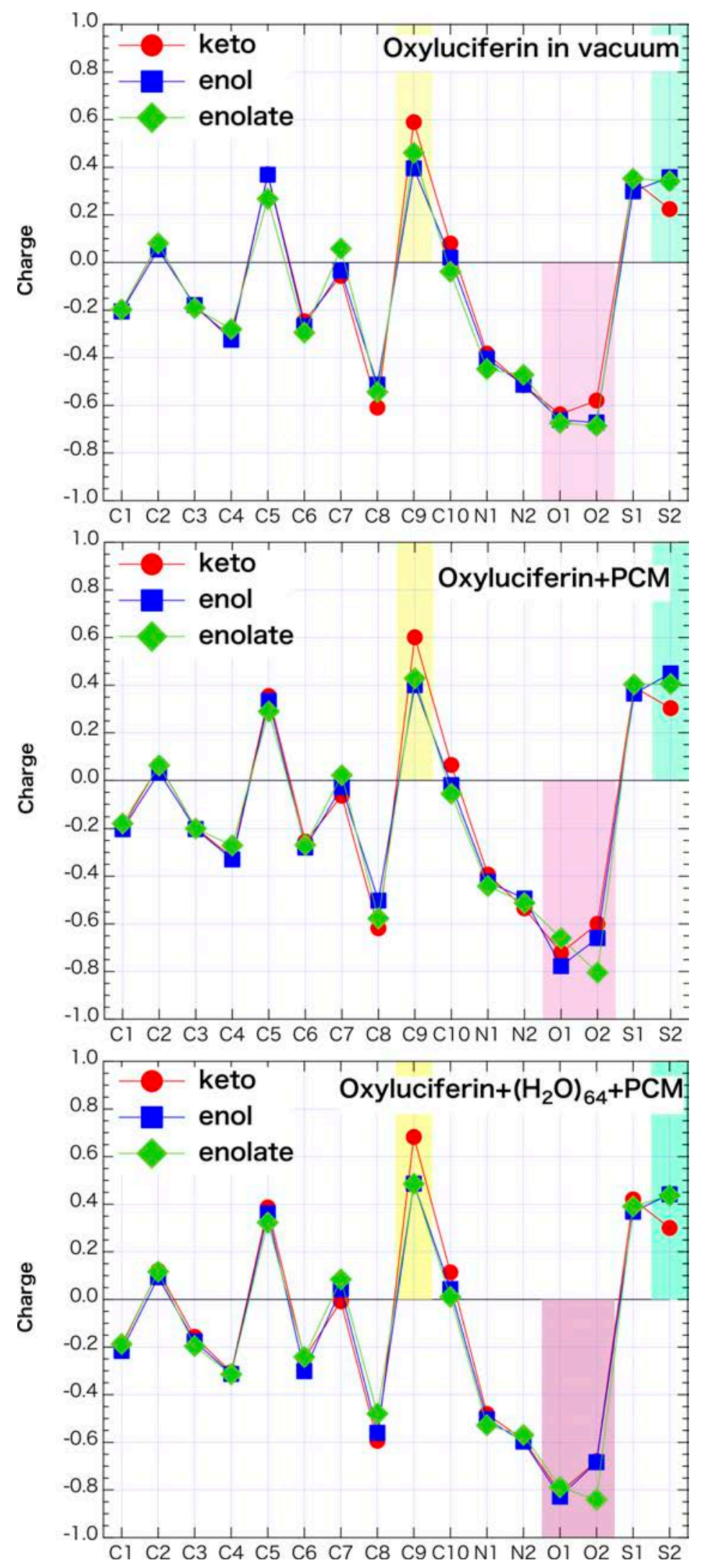

Figure S2. Natural population analysis for oxyluciferin in vacuum, in PCM, and in aqueous solution. 

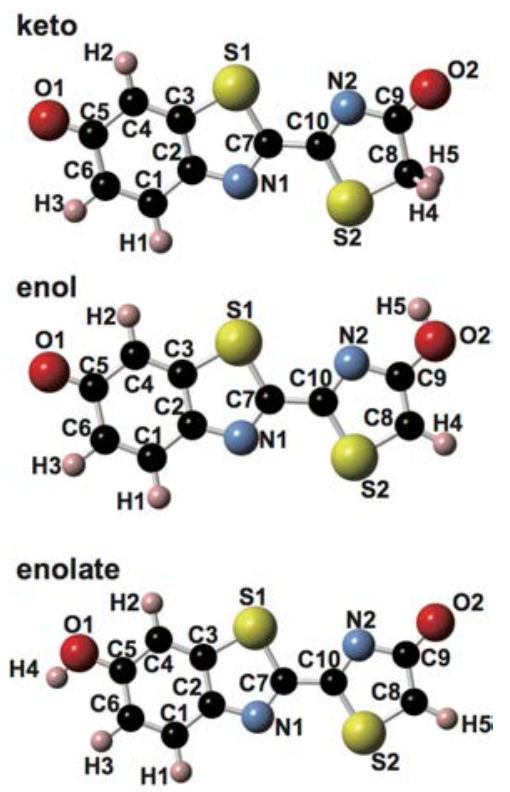

Figure S3. Atomic geometries optimized for keto, enol, and enolate. Each atom is labeled here for the following discussion.
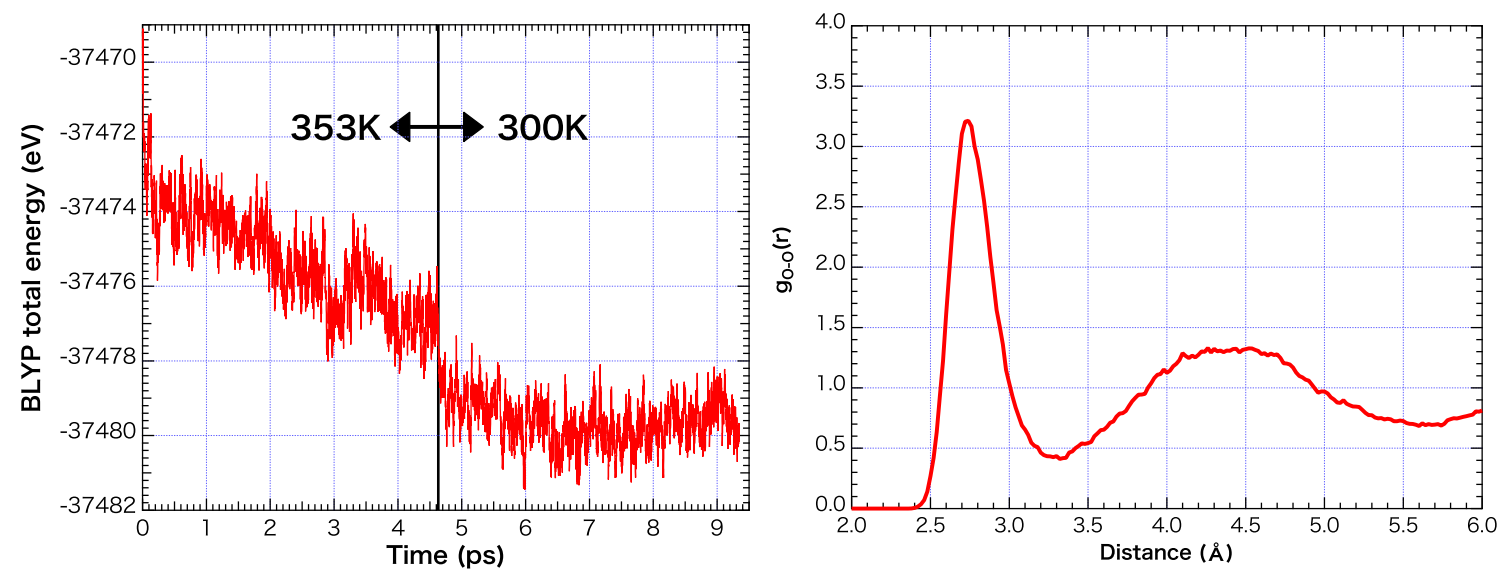

Figure S4. BLYP total energy simulated for $\left(\mathrm{H}_{2} \mathrm{O}\right)_{80}$ and radial distribution function between oxygen atoms. 


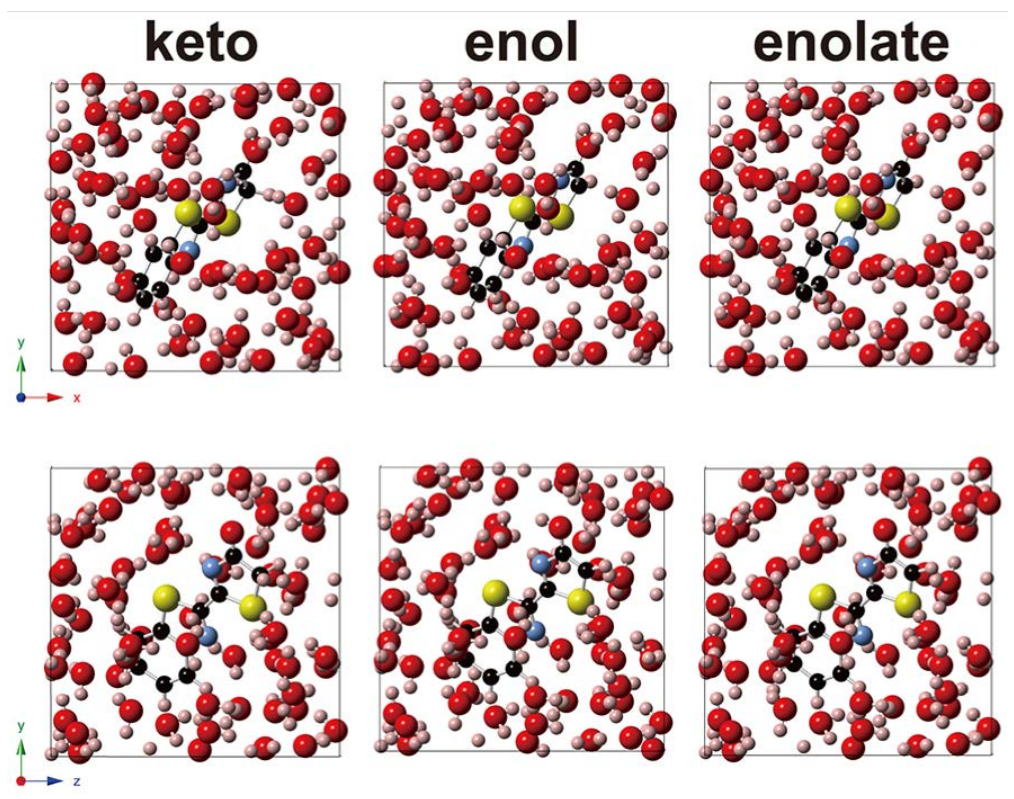

Figure S5. Initial atomic geometries of keto-, enol-, and enolate-type oxyluciferin anion in $\left(\mathrm{H}_{2} \mathrm{O}\right)_{64}$. 


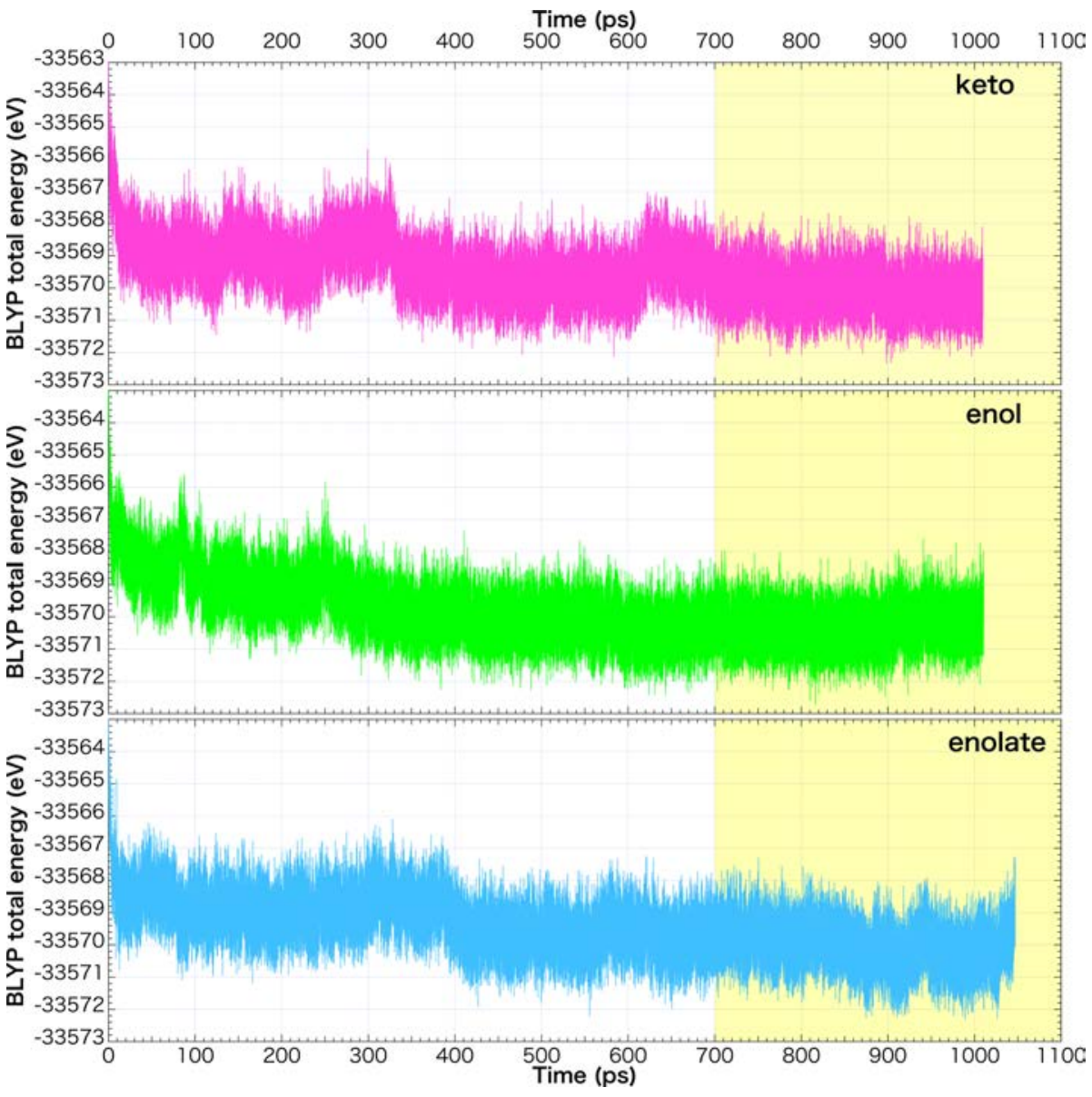

Figure S6. BLYP total energies in eV. 
Table S2. Bond distance in $\AA$.

\begin{tabular}{cccccccccc}
\hline \hline & \multicolumn{3}{c}{ keto } & \multicolumn{3}{c}{ enol } & \multicolumn{3}{c}{ enolate } \\
label & vacuum & PCM & solution & vacuum & PCM & solution & vacuum & PCM & solution \\
\hline C8-C9 & 1.56 & 1.55 & 1.54 & 1.37 & 1.38 & 1.38 & 1.44 & 1.42 & 1.40 \\
\hline O1-C5 & 1.27 & 1.28 & 1.32 & 1.27 & 1.29 & 1.34 & 1.40 & 1.38 & 1.39 \\
O2-C9 & 1.23 & 1.24 & 1.25 & 1.38 & 1.37 & 1.36 & 1.26 & 1.28 & 1.32 \\
\hline N1-C2 & 1.35 & 1.35 & 1.36 & 1.36 & 1.37 & 1.39 & 1.38 & 1.38 & 1.39 \\
N1-C7 & 1.33 & 1.33 & 1.33 & 1.32 & 1.32 & 1.32 & 1.32 & 1.31 & 1.32 \\
N2-C9 & 1.38 & 1.38 & 1.38 & 1.36 & 1.37 & 1.38 & 1.43 & 1.42 & 1.40 \\
N2-C10 & 1.32 & 1.33 & 1.34 & 1.33 & 1.33 & 1.33 & 1.31 & 1.32 & 1.33 \\
\hline S1-C3 & 1.77 & 1.77 & 1.75 & 1.78 & 1.77 & 1.75 & 1.76 & 1.76 & 1.75 \\
S1-C7 & 1.81 & 1.80 & 1.79 & 1.81 & 1.81 & 1.78 & 1.82 & 1.81 & 1.79 \\
S2-C8 & 1.83 & 1.84 & 1.84 & 1.76 & 1.75 & 1.74 & 1.73 & 1.74 & 1.72 \\
S2-C10 & 1.82 & 1.80 & 1.77 & 1.79 & 1.78 & 1.75 & 1.78 & 1.77 & 1.75 \\
\hline \hline
\end{tabular}

Table S3. Averaged number of hydrogen bonds.

\begin{tabular}{cccc}
\hline \hline label & keto & enol & enolate \\
\hline $\mathrm{N} 1$ & 0.49 & 0.93 & 0.98 \\
$\mathrm{~N} 2$ & 0.97 & 1.33 & 1.05 \\
$\mathrm{O} 1$ & 2.93 & 2.97 & 0.93 \\
$\mathrm{O} 2$ & 1.41 & 0.48 & 2.96 \\
$\mathrm{H} 4$ & - & - & 0.92 \\
$\mathrm{H} 5$ & - & 0.97 & - \\
\hline \hline
\end{tabular}



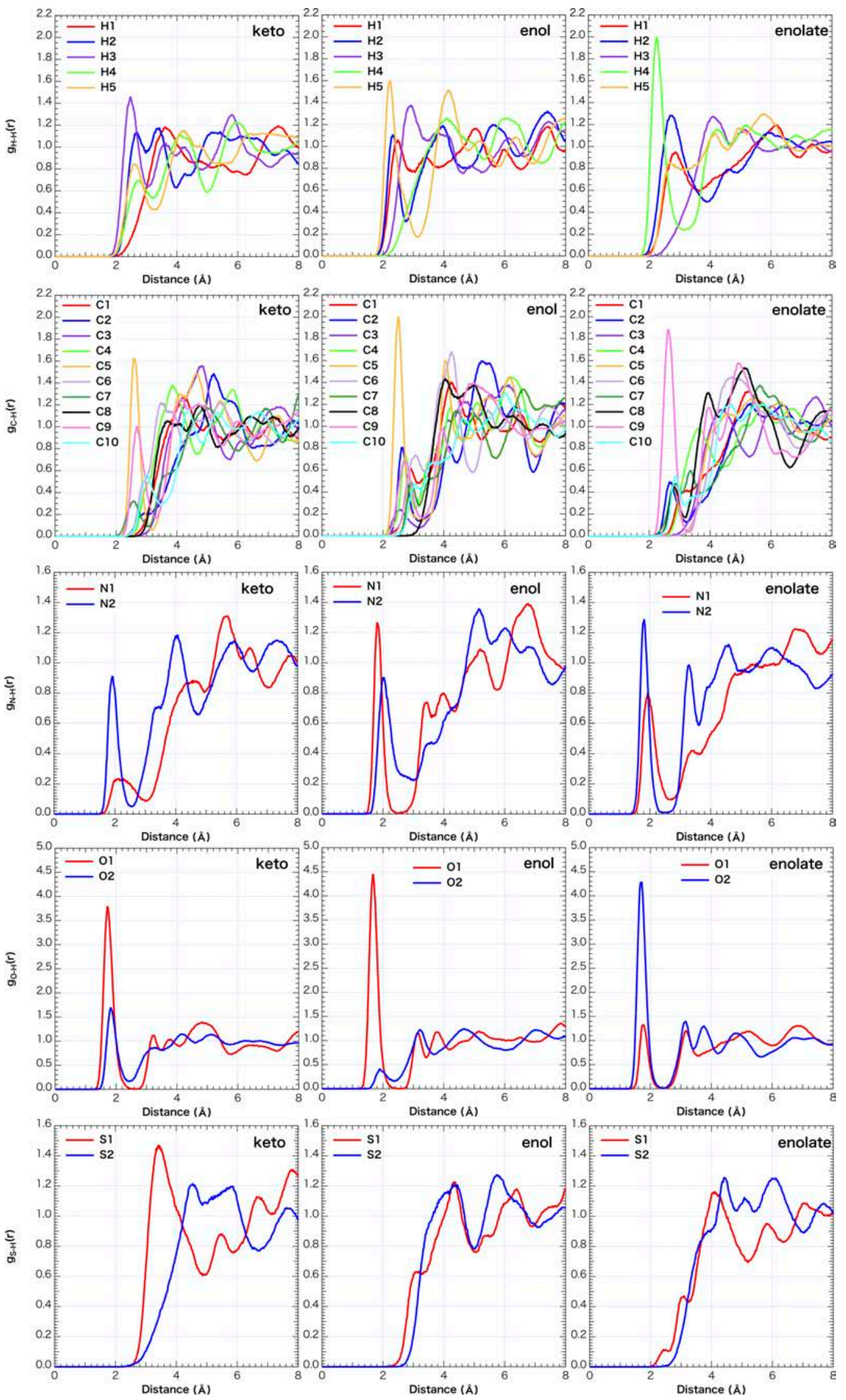

Figure S7. Radial distribution function with hydrogen atoms. 

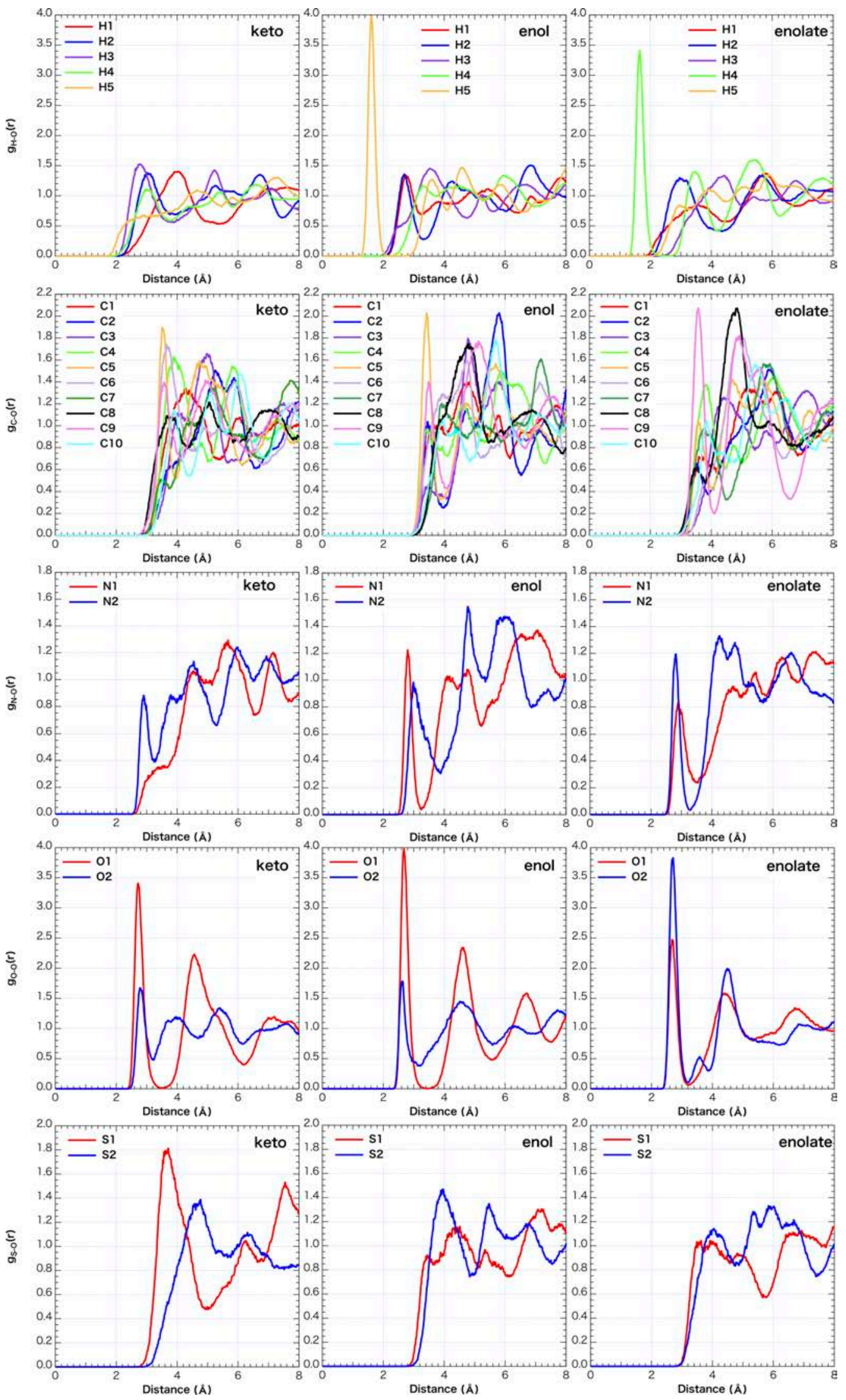

Figure S8. Radial distribution function with oxygen atoms 

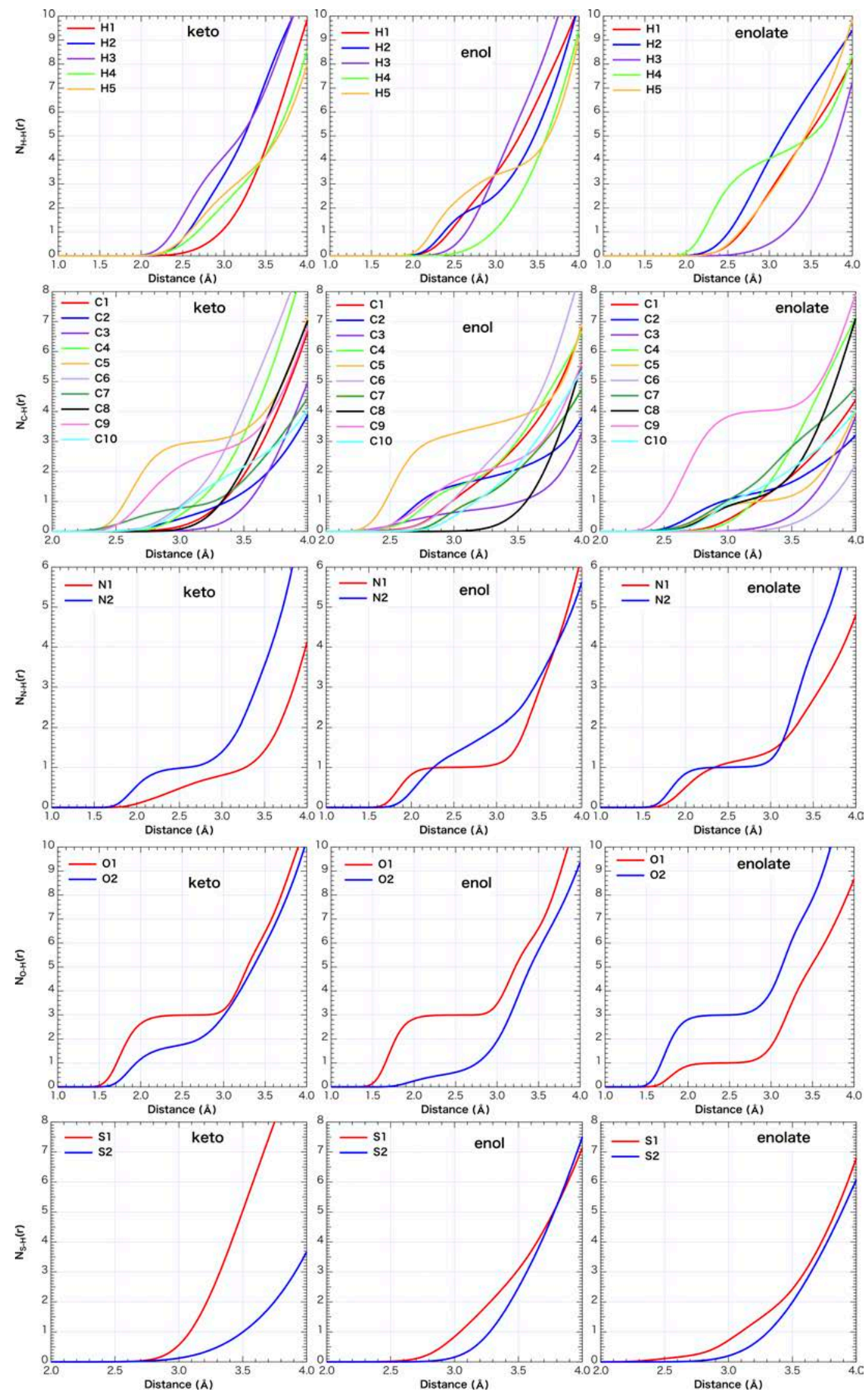

Figure S9. Coordination number with hydrogen atoms. 

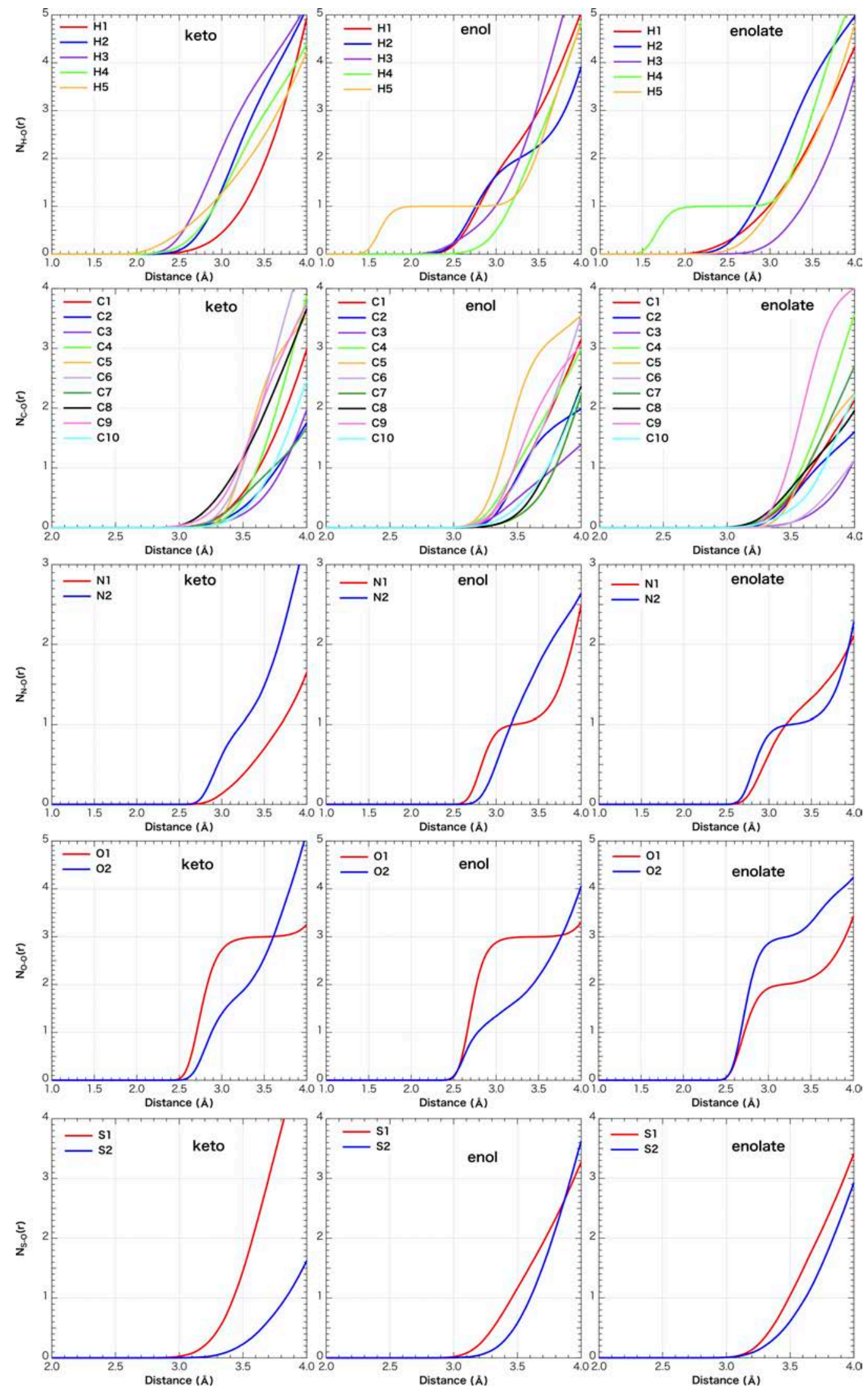

Figure S10. Coordination number with oxygen atoms. 


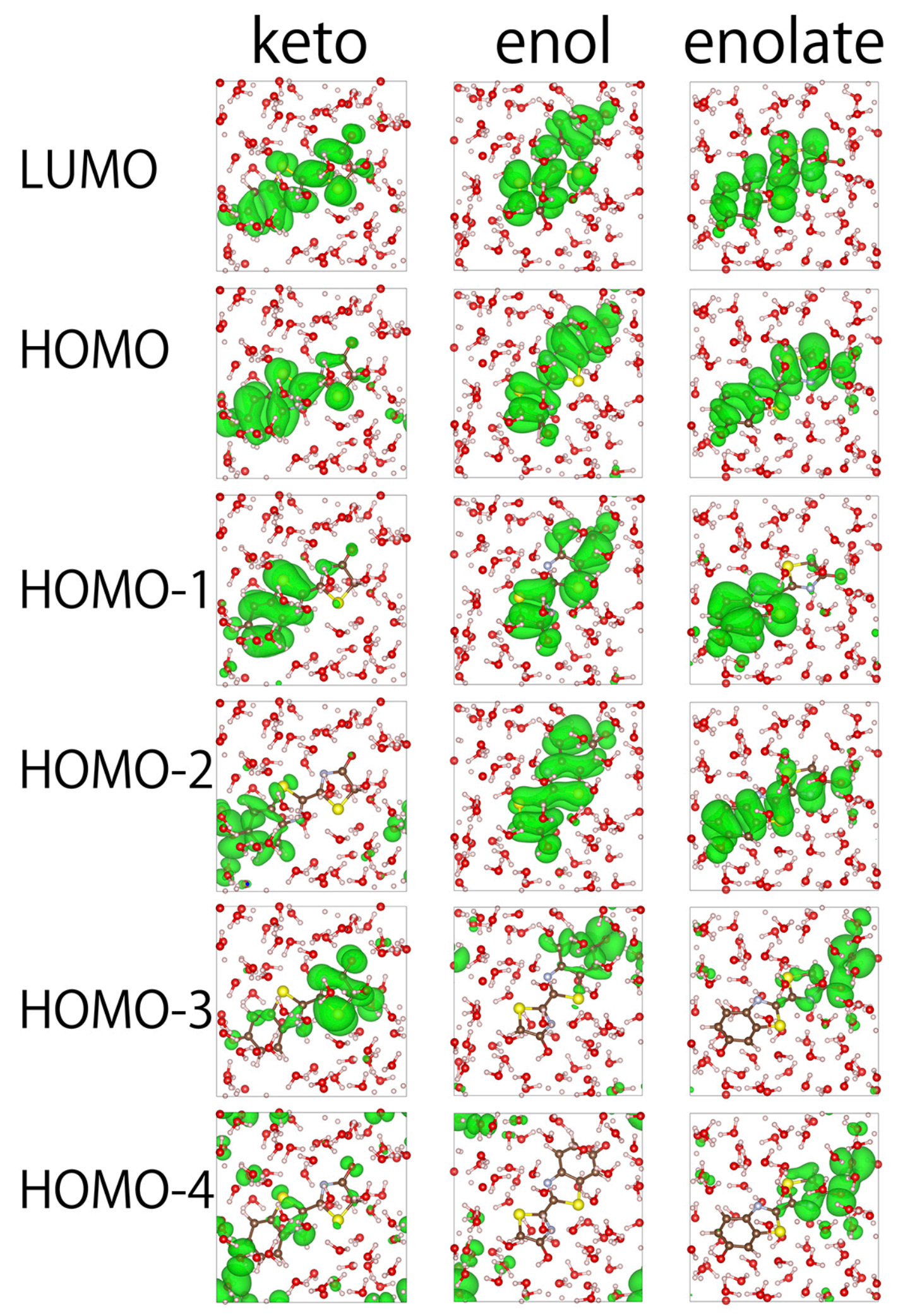

Figure S11. BLYP Kohn-Sham wave functions at some occupied and LUMO levels calculated for the snapshot randomly selected from MD results. Here, the LUMO level is set to be $0 \mathrm{eV}$. 


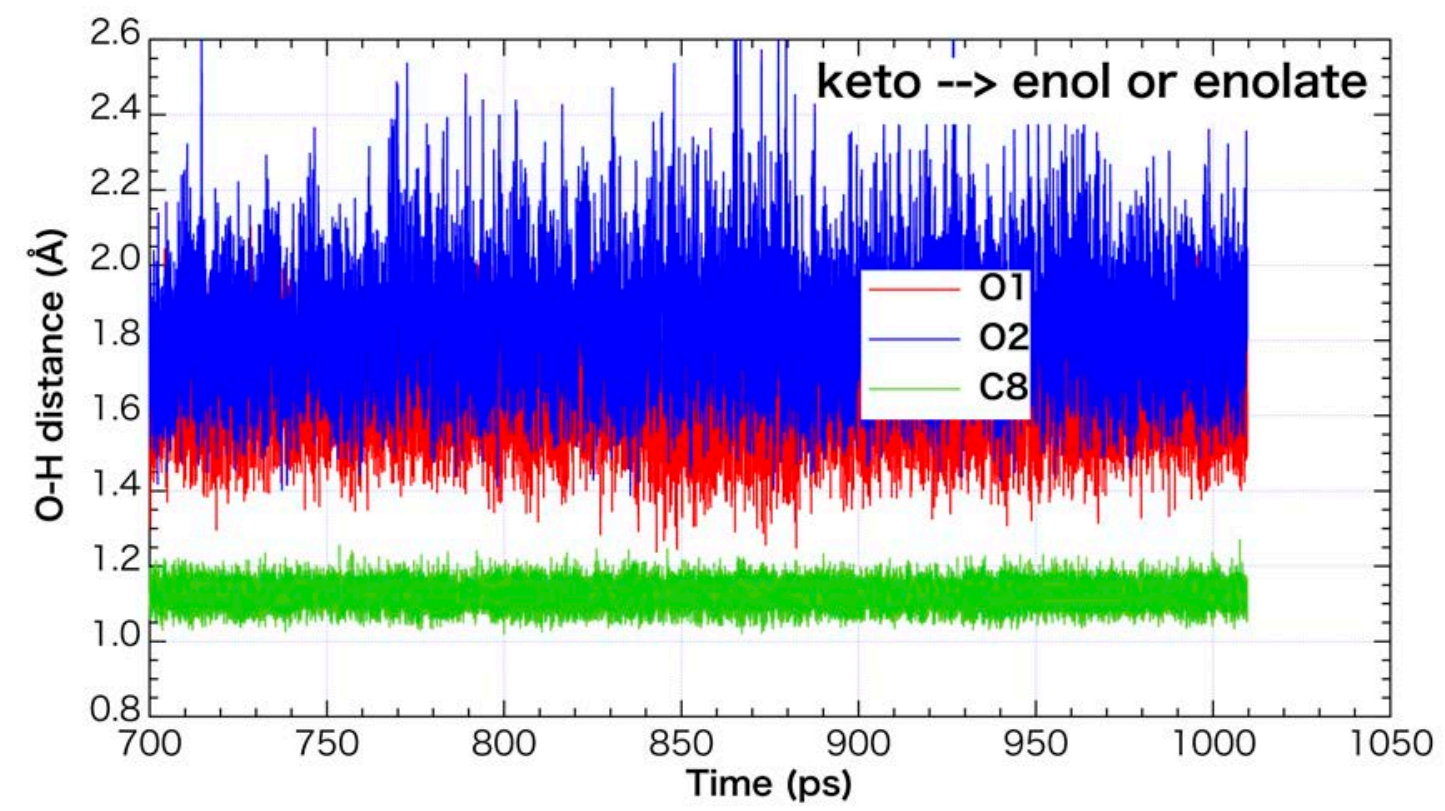

Figure S12. Chemical bond distance between keto-type oxyluciferin (C8) and oxyluciferin (H4 or H5) and hydrogen bond distance between keto-type oxyluciferin (O1 and $\mathrm{O2}$ ) and the nearestneighbor water molecule $(\mathrm{H})$. The label of atom is given in Fig. S3.

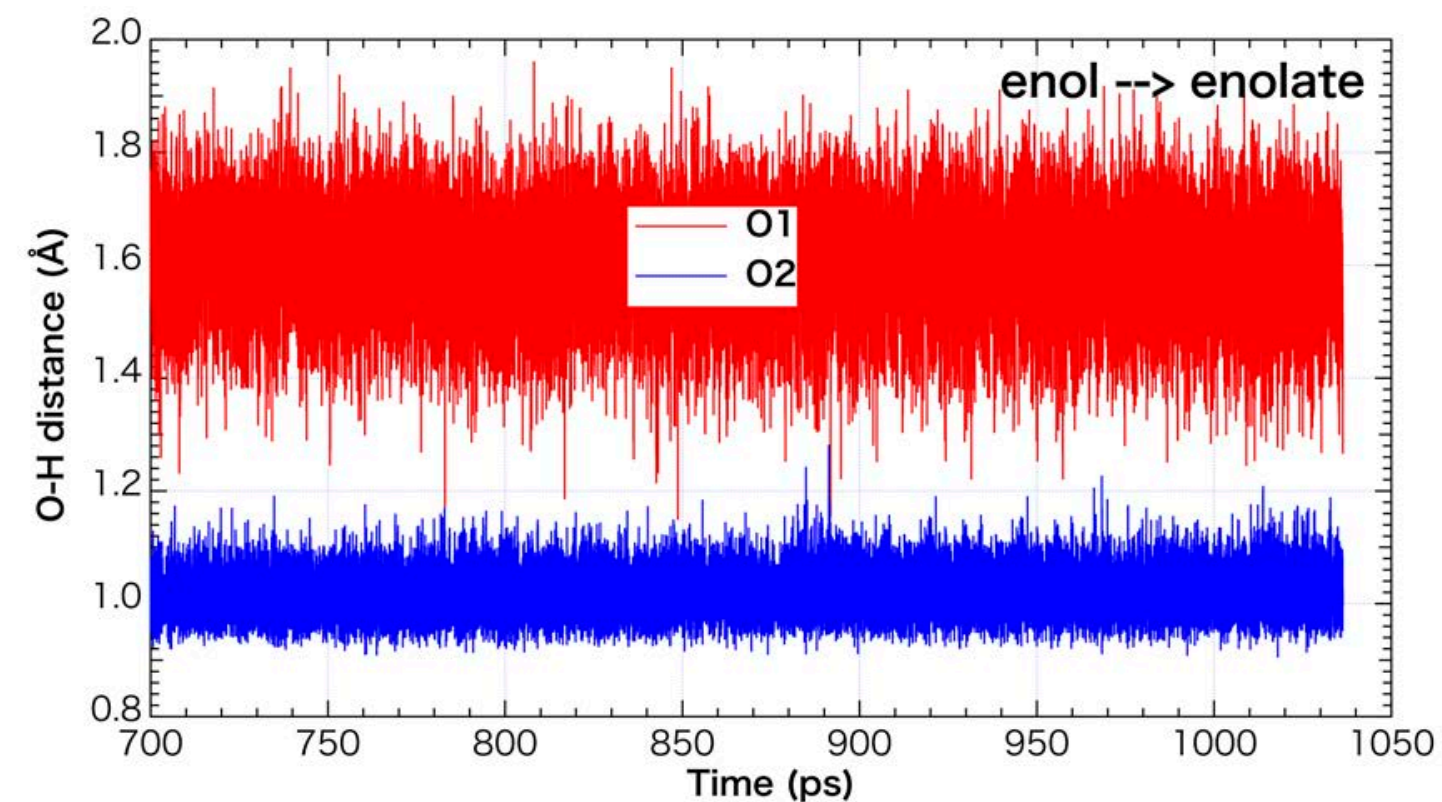

Figure S13. Chemical bond distance between enol-type oxyluciferin (02) and the enol-type (H5) and hydrogen bond distance between enol-type oxyluciferin (O1) and the nearestneighbor water molecule $(\mathrm{H})$. The label of atom is given in Fig. S3. 


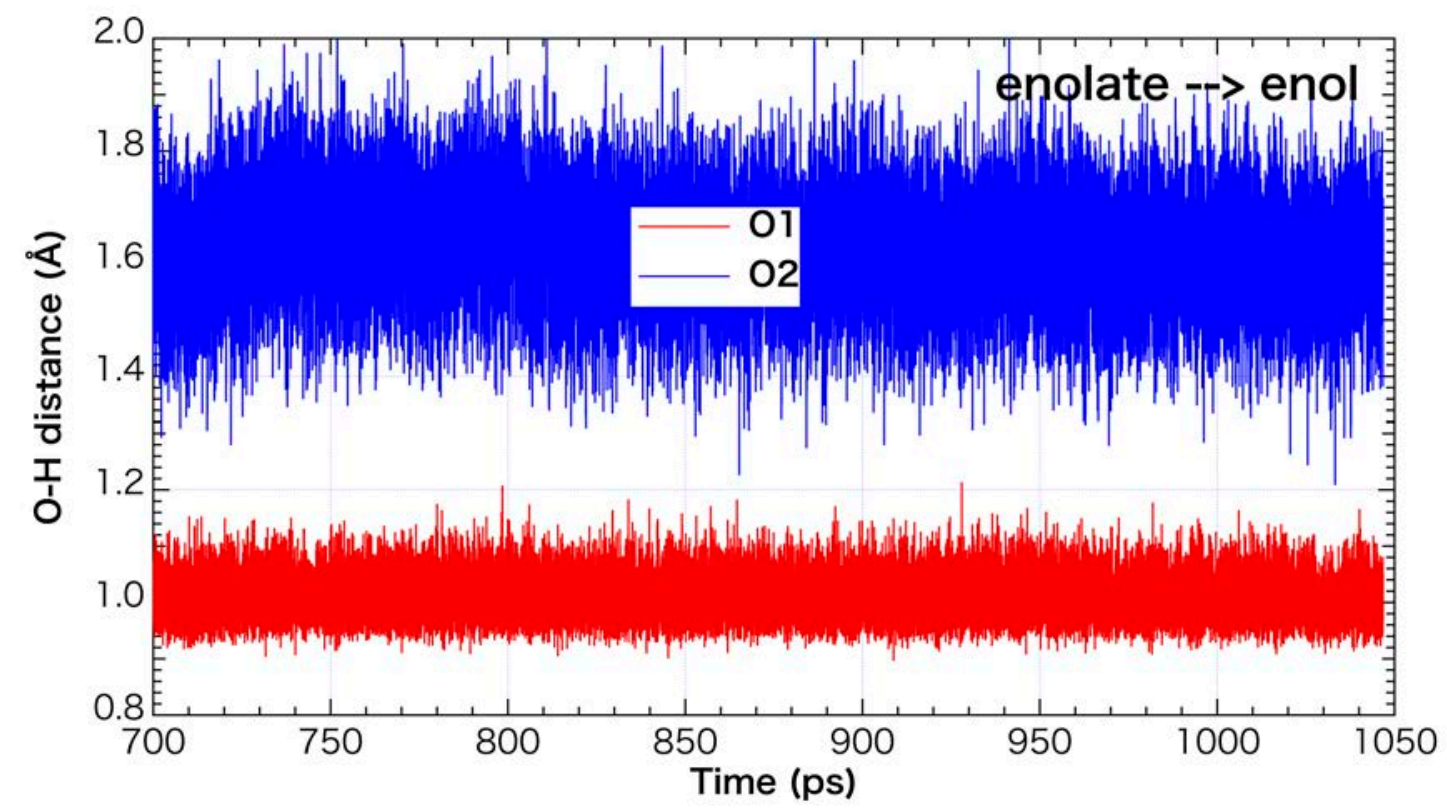

Figure S14. Chemical bond distance between enolate-type oxyluciferin (01) and the enol-type (H4) and hydrogen bond distance between enolate-type oxyluciferin (O2) and the nearestneighbor water molecule $(\mathrm{H})$. The label of atom is given in Fig. S3. 